\title{
Tubeless PCNL - Contemporary Analysis of Indications, Safety and Efficacy
}

\author{
Dr. Ramaiah Keshavamurthy ${ }^{1}$, Dr. Sachin Dharwadkar ${ }^{2 *}$, Dr. Sumit Kumar ${ }^{2}$, Dr. Vilvapathy \\ Senguttuvan Karthikeyan ${ }^{2}$ \\ ${ }^{I}$ Professor and Head, Department of Urology, Institute of Nephrourology, Victoria Hospital Campus, \\ Bangalore 560002, India \\ ${ }^{2}$ Senior Resident, Department of Urology, Institute of Nephrourology, Bangalore 560002, India
}

*Corresponding Author: Dr. SachinDharwadkar, Senior Resident, Department of Urology, Institute of Nephrourology, Bangalore 560002, India, E-mail: drsachinvd@gmail.com

\begin{abstract}
Introduction: Percutaneous nephrolithotomy (PCNL) has been modified to use of small diameter tubes, moving towards 'totally tubeless' PCNL. We assessed the indications, safety and efficacy of tubeless PCNL in comparison with standard PCNL in the contemporary era.
\end{abstract}

Methods: Patients undergoing PCNL in a tertiary care center from January 2013 to September 2015 were enrolled for the study. Stone free status, length of hospitalization (LOH) and complications especially bleeding were compared between tubeless and standard PCNL.

Result: Tubeless PCNL contributed to $31.8 \%$ (273/858) of our patients. Operation time (OT), larger stone size, multiple stones or staghorn and diverticular stones were significantly contributory to placement of a nephrostomy tube. The median LOH (5 vs 3 days) was significantly $(p<0.001)$ higher in standard PCNL. SFS was significantly $(p<0.001)$ higher in tubeless (97.6\%) than that in the standard $(70.6 \%)$ PCNL. Bleeding rates were similar in both groups.

Conclusion: Tubeless PCNL is an effective and safe procedure for treatment of renal stones in select patients. Placement of a nephrostomy is safer in patients with bleeding, pelvic perforation and provides another chance to maximise clearance of residual stones.

Keywords: Percutaneous nephrolithotomy; Tubeless; Standard; CROES-Clavien system

\section{INTRODUCTION}

Percutaneous nephrolithotomy (PCNL) was developed in 1970 s as an adjunct to open surgery for renal calculi (1). Routine use of a nephrostomy was challenged in 1997and there has been a trend towards tubeless PCNL (2). Advantages include shorter length of hospitalization $(\mathrm{LOH})$, reduced analgesic requirements and a smoother postoperative recovery. Tubeless PCNL has been reported to be safe in renal anomalies and atypical renal anatomy as well (3). In patients with hemorrhage, standard PCNL with placement of nephrostomy is still an accepted practice. We report the indications, safety and efficacy of tubeless PCNL in the contemporary era and in whom standard PCNL is still a valid option.

\section{METHODS}

Patients undergoing PCNL for renal calculi in a tertiary care referral urological centre between
January 2013 and September 2015 were included. Institutional research and ethics committee approval were obtained. Preoperative workup included hemoglobin, serum creatinine, urine routine and microscopy, urine culture and sensitivity, ultrasound (US) kidneys, ureter and bladder (KUB) and plain X-ray (XR) KUB. An intravenous pyelogram or unenhanced computed tomogram KUB was performed based on serum creatinine and history of contrast allergy. Stone free status (SFS) was defined as absence of stone fragments more than $4 \mathrm{~mm}$ on US or XR KUB.

\subsection{Patient Workup}

Data included age, gender, comorbidities and past surgical history. Stone characteristics included stone location, dimensions $(\mathrm{mm})$, number, presence of staghorn (partial or complete) calculus, Guy's stone score (GSS), anatomy of the kidneys and Hounsfeld units 
(HU) (4). Intra operative parameters included access (supra/ infracostal), number of punctures, calyx accessed, tract dilation, operative time (OT), postoperative placement of nephrostomy, double $\mathbf{J}$ stent or ureteral catheter. Postoperative variables assessed included LOH, SFS, residual stone status, anal gesic requirements and complications based on CROES-Clavien system $(5,6)$.

\subsection{Operative Procedure}

PCNL was performed by a standard method und er general anesthesia. Cystoscopy (Karl Storz Endoscopy, Tuttlingen, Germany) and ureteral catheterization was performed with $5 \mathrm{~F}$ openended $70 \mathrm{~cm}$ ureteral catheter. Retrograde pyelography was done after patient was turned prone and appropriate calyx was punctured using $18 \mathrm{G} / 15 \mathrm{~cm}$ diamond tipped needle by Bull's eye technique under image intensifier. Serial Teflon and metal tract dilation were done under fluoros copy guidance to $26 \mathrm{~F}$ or $30 \mathrm{~F}$ and rigid $(22 \mathrm{~F}$ or $26 \mathrm{~F})$ nephroscope(Karl Storz Endoscopy, Tuttlin gen, Germany) was used for pneumatic lithotripsy (NidhiLithDigi, NidhiMeditech Systems, India).Operative time (OT) from access to placement of nephrostomy/ closure of access was calculated. Tubeless PCNL was performed based on surgeon discretion and intraoperative factors. Ureteric catheter and nephrostomy tube were removed after 48 hours if the patient had no hem aturia or fever. Stents if placed were removed after 1 month.

\subsection{Statistical Analysis}

Data was tabulated using Microsoft Excel 2007 and analyzed using SPSS v20 (IBM Corp, Armonk, NY, USA). A $p$ value $<0.05$ was considered as statistically significant. Data was represented as frequency, percentages, mean or median based on the characteristics of variables. Continuous variables were compared using Students't-test (two tailed, independent) or Mann Whitney $U$ test based on normality of data. For categorical variables, Chi squared or Fisher's exact test was used. Receiver operating characteristic curve analysis was done to identify cutoff values of OT and stone size for predicting successful tubeless PCNL application. Binomial logistic regression analysis was performed to identify factors leading to placement of a nephrostomy tube.

\section{RESULTS}

A total of 858 patients underwent PCNL during the study period with 273 (31.81\%) of them undergoing tubeless PCNL. The baseline patient characteristics like age, gender, prior surgery, renal function and side were comparable among tubeless and standard PCNL groups (Table 1).

Table1. Baseline characteristics

\begin{tabular}{|l|l|l|l|}
\hline \multicolumn{1}{|c|}{ Characteristics } & \multicolumn{1}{c|}{ Tubeless n (\%) } & \multicolumn{1}{c|}{ Nephrostomy n (\%) } & P value \\
\hline Age (Mean \pm SD) & $41.9 \pm 13.6$ & $43.7 \pm 13.3$ & 0.071 \\
\hline Gender & $171(29.9)$ & $400(70.1)$ & 0.097 \\
\hline Male & $102(35.5)$ & $185(64.5)$ & \\
\hline Female & $60(34.9)$ & $112(65.1)$ & 0.334 \\
\hline Previous stone surgery & $21(27.6)$ & $55(72.4)$ & 0.412 \\
\hline Renal failure & $1.12 \pm 0.76$ & $1.2 \pm 1.11$ & 0.231 \\
\hline Creatinine (Mean \pm SD) & & & 0.171 \\
\hline Side & $143(29.4)$ & $343(70.6)$ & \\
\hline Right & $130(35)$ & $242(65)$ & \\
\hline Left & & & \\
\hline
\end{tabular}

\subsection{Stone Characteristics}

The stone size was significantly higher in patients undergoing standard PCNL $(\mathrm{p}<0.001)$ with a higher incidence of GSS 3 and 4 and stone bur den $(p<0.001)$.
Nephrostomy was placed in more of upper calcyceal punctures $(61 \%$ vs $39 \%$; $p<0.001)$ and staghorn stones $(82.5 \%$ vs $17.5 \%$; $\mathrm{p}<0$. 001). The distribution of anomalous kidneys and stone density did not affect nephrostomy tube placement (Table 2).

Table2. Stone characteristics

\begin{tabular}{|l|l|l|l|}
\hline \multicolumn{1}{|c|}{ Characteristics } & \multicolumn{1}{|c|}{ Tubeless n (\%) } & \multicolumn{1}{c|}{ Nephrostomy n (\%) } & P value \\
\hline Stone size (Mean \pm SD) & $20.1 \pm 6.1$ & $23.5 \pm 8.1$ & $<0.001$ \\
\hline Stone burden (Mean \pm SD) & $258.3 \pm 169$ & $361.3 \pm 263.1$ & $<0.001$ \\
\hline Location of stone & $171(39)$ & $267(61)$ & $<0.001$ \\
\hline Upper & $35(36.8)$ & $60(63.2)$ & \\
\hline Lower & $67(20.6)$ & $258(79.4)$ & \\
\hline Multiple/ diverticulum &
\end{tabular}


Tubeless PCNL - Contemporary Analysis of Indications, Safety and Efficacy

\begin{tabular}{|l|l|l|l|}
\hline Staghorn calculus & $20(17.5)$ & $94(82.5)$ & $<0.001$ \\
\hline Hydronephrosis & $235(31.9)$ & $502(68.1)$ & 0.916 \\
\hline Anomalous kidneys & $5(23.8)$ & $16(76.2)$ & 0.425 \\
\hline GSS (Mean \pm SD) & $1.47 \pm 0.89$ & $1.88 \pm 1.1$ & $<0.001$ \\
\hline HU (Mean \pm SD) & $1010.3 \pm 237.7$ & $1008 \pm 257$ & 0.917 \\
\hline
\end{tabular}

*: GSS-Guy' stone score; HU-Hounsfeld units

\subsection{Intraoperative Characteristics}

In non-lower calyx punctures $(\mathrm{p}=0.014)$, larger diameter tracts $(\mathrm{p}=0.007)$ and multiple tracts $(\mathrm{p}$ $=0.001)$, there was a higher tendency of nephrostomy tube placement. Supracostalaccess did not contribute to nephrostomy tube placement (Table 3). Based on ROC analysis, for a stone size $\geq 19.5 \mathrm{~mm}$, standard PCNL was necessary (AUC: $0.632 ; 65.3 \%$ sensitivity and $54.2 \%$ specificity).
A cut off OT of $\geq 72.5$ min was able to predict standard PCNL with $65.5 \%$ sensitivity and 61.9\% sensitivity (AUC: 0.690) (Fig. 1a, b).

These factors also contributed to placement of a nephrostomy tube and were not sole indications. Based on multivariate logistic regression, OT, larger stone size, multiple stones/ staghorn and diverticular stones significantly contributed to placement of a nephrostomy tube (Table 4).

Table3. Intraoperative characteristics

\begin{tabular}{|c|c|c|c|}
\hline Characteristics & Tubeless & Nephrostomy & P value \\
\hline Operative time (Mean \pm SD) & $67.3 \pm 21.1$ & $83.2 \pm 25.7$ & $<0.001$ \\
\hline \multicolumn{3}{|l|}{ Site of puncture $\mathrm{N}(\%)$} & \multirow[t]{3}{*}{0.014} \\
\hline Lower pole & $122(44.7)$ & $243(41.5)$ & \\
\hline Non lower pole & $151(55.3)$ & $342(58.5)$ & \\
\hline \multicolumn{3}{|l|}{ Access N (\%) } & \multirow[t]{3}{*}{0.38} \\
\hline Supracostal & $94(34.4)$ & $245(41.9)$ & \\
\hline Infracostal & $179(65.6)$ & $340(58.1)$ & \\
\hline \multicolumn{3}{|l|}{ Tract size N (\%) } & \multirow[t]{3}{*}{0.007} \\
\hline $26 \mathrm{~F}$ & $159(58.3)$ & $274(46.7)$ & \\
\hline $30 \mathrm{~F}$ & $114(41.8)$ & $311(53.2)$ & \\
\hline No. of punctures(Mean \pm SD) & $1(1)$ & $1(0)$ & 0.001 \\
\hline
\end{tabular}
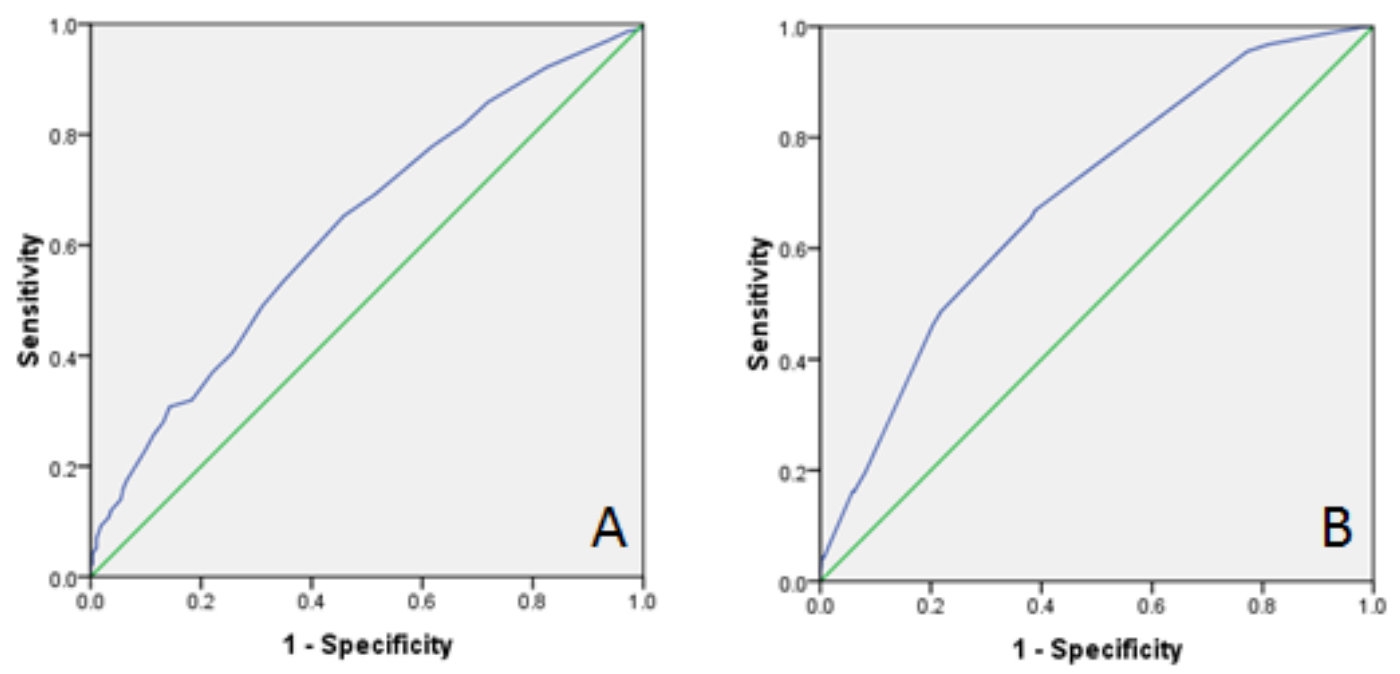

Fig1. Receiver operating characteristic curve analysis to predict feasibility of tubeless percutaneous nephrolithotomy: A) Stone size; B) Operation time.

Table4. Factors predicting placement of nephrostomy - Multivariate analysis

\begin{tabular}{|c|c|c|}
\hline Factors & Adjusted OR (OR, 95\% CI) & Pvalue \\
\hline Duration of procedure $>72.5$ minutes & $2.722(1,989,3.724)$ & $<0.001$ \\
\hline Supracostal approach & $1.187(0.862,1.634)$ & 0.293 \\
\hline Stone size $>19.5 \mathrm{~mm}$ & $1.631(1.172,2.268)$ & 0.004 \\
\hline Past surgery & $0.902(0.619,1.314)$ & 0.589 \\
\hline Hydronephrosis & $0.682(0.428,1.087)$ & 0.107 \\
\hline Multiple stone/ Diverticulum & $1.797(1.251,2.583)$ & 0.002 \\
\hline
\end{tabular}




\subsection{Postoperative Characteristics}

Whenever the stone clearance was not adequate, there was a tendency to place a nephrostomy tube. In $30.6 \%(179 / 585)$ patients of standard PCNL, residual stones were seen with a mean of $2.72 \mathrm{~cm}$ and $73(12.5 \%)$ patients underwent relook PCNL $(\mathrm{p}<0.001)$ for increasing SFS. There was a trend towards higher CCS complications in standard PCNL $(\mathrm{p}<0.001)$.
The median LOH (5 vs 3 days) was significantly $(p<0.001)$ higher in standard PCNL which were possibly due to complex stones and complications (Table 5).

SFS in patients in tubeless (97.6\%) group was significantly $(\mathrm{p}<0.001)$ higher than that of the standard PCNL $(70.6 \%)$ group. Bleeding rates were similar in both groups (Table 5).

Table5. Post Procedure characteristics

\begin{tabular}{|c|c|c|c|}
\hline Characteristics & Tubeless n (\%) & Nephrostomy n (\%) & P value \\
\hline Residual stones $>4 \mathrm{~mm}$ & $9(3.3)$ & $179(30.6)$ & \multirow[t]{2}{*}{$<0.001$} \\
\hline Residual size (Mean \pm SD) & $0.22 \pm 1.3$ & $2.72 \pm 4.58$ & \\
\hline Hemoglobin drop (g/dL) & $1.1 \pm 1.2$ & $1.3 \pm 0.9$ & 0.542 \\
\hline $\mathrm{LOH}(\mathrm{Median} \pm \mathrm{SD})$ & $3(1)$ & $5(2)$ & $<0.001$ \\
\hline Complications & $28(10.3)$ & $210(36.1)$ & $<0.001$ \\
\hline \multicolumn{4}{|c|}{ CROES-Clavien classification } \\
\hline 0 & $244(89.4)$ & $374(64)$ & \multirow[t]{5}{*}{$<0.001$} \\
\hline 1 & $20(7.3)$ & $120(20.5)$ & \\
\hline 2 & $7(2.6)$ & $60(10.3)$ & \\
\hline 3 & $2(0.7)$ & $26(4.5)$ & \\
\hline 4 & 0 & $4(0.7)$ & \\
\hline Relook PCNL & $2(0.2)$ & $73(12.5)$ & $<0.001$ \\
\hline
\end{tabular}

*: LOH - Length of hospitalization

\section{DISCUSSION}

Since its introduction, the technique of PCNL has constantly been improvised. With a better understanding of anatomy of kidney, operative procedure of PCNL and smaller diameter tracts, bleeding as a complication has reduced. Bellman et al identified that tubeless PCNL is feasible and has the advantages of shorter $\mathrm{LOH}$, early convalescence, reduced analgesic requirements and cost-savings which was replicated in other studies $(2,3,7,8)$. Wickham et al proved that it is safe to avoid a stent or ureteral catheter in tubeless PCNL (7).Safety of tubeless PCNL has been proved in patients with renal anomalies including horseshoe, malrotated and ectopic kidneys and in older patients aged beyond 60 years $(3,8)$.

Placement of a nephrostomy tube or ureteral catheter may increase the risk of postoperative pain and morbidity (9-12). When the nephrostomy was avoided, it was observed that complications were comparable to standard PCNL and that tubeless PCNL was safe and costeffective (13). Despite its potential advantages, widespread acceptance of tubeless PCNL has been slow (14). PCNL is a challenging operation with a complication rate of $1.1-7 \%$ in experienced hands. The main problem is hemorrhage that can happen during any stage of the procedure. Placing a nephrostomy may help avoid this complication and provides the opportunity for second look PCNL or percutaneous chemolitholysis $(9,15)$. Despite continual technical refinements, hemorrhage is still a problem as reported in a meta-analysis and placement of a nephrostomy has been found to reduce it (15).

We performed tubeless PCNL safely in patients with anomalous kidneys, staghorn or complex renal calculi, multiple tracts and supra costal approach. We found that tubeless PCNL is safe in patients with no evidence of perforation of renal pelvis, minimal intraoperative bleeding and in patients in whom there was no need of a relook PCNL as also reported by Tirtayasa (9). We observed that multiple tract PCNL was not a contraindication for tubeless PCNL in our experience.

Cost of performing tubeless PCNL was reported to be significantly lesser than standard PCNL in a study done by Choi et al $(9,15)$.Bleeding has always been a pointer against tubeless PCNL. In a large report of 1000 tubeless PCNL with 17\% patients having staghorncalculi, custom-made electrode probe was used to cauterize bleeding points. In patients with uncontrolled bleed, oxidized regenerated cellulose strips were used to pack and tamponade the renal parenchyma (16). The cost-effectiveness of this approach and its efficacy needs to be assessed and could possibly aid in avoiding a nephrostomy for fear of bleeding if there is no other contraindication. 
In a review, favorable outcomes were seen in a select group of patients undergoing tubeless PCNL with stone burden $<3 \mathrm{~cm}$, single access, no significant bleeding, perforation and residual calculi and had advantages of reduced postoperative pain and hospital stay (17).

\section{CONCLUSION}

In the contemporary era, tubeless PCNL can be safely performed in patients with reasonable intraoperative stone free rate. In patients in whom bleeding is a problem, placement of nephrostomy is safe. Judicious application of tubeless PCNL can aid in quick recovery of patient enjoying the benefits of shorter $\mathrm{LOH}$, less cost and reduced pain.

\section{REFERENCES}

[1] Fernström I, Johansson B. Percutaneous pyelo lithotomy. A new extraction technique. Scand J UrolNephrol 1976; 10(3):257-9.

[2] Bellman GC, Davidoff R, Candela J, Gerspach J, Kurtz S, Stout L.Tubeless percutaneous renal surgery. JUrol 1997; 157(5):1578-82.

[3] Aghamir SM, Mohammadi A, Mosavibahar SH, MeysamieAP. Totally tubeless percutaneousn ephro lithotomy in renal anomalies .JEndo urol 2008; 22(9):2131-4.

[4] Thomas K, Smith NC, Hegarty N, Glass JM. The Guy's stone score--grading the complexity of percutaneous nephrolithotomy procedures. Urology2011; 78(2):277-81.

[5] de la Rosette JJ, Opondo D, Daels FP, Giusti G, Serrano A, Kandasami SV, et al. Categorisation of complications and validation of the Clavien score for percutaneous nephrolithotomy. EurUrol 2012; 62:246-55.

[6] Smith A, Averch TD, Shahrour K, Opondo D, Daels FP, Labate G, et al. CROES PCNL Study Group. A nephrolithometricnomogramto predict treatment success of percutaneous nephrolithotomy. J Urol 2013; 190:149-56.
[7] Wickham JE, Miller RA, Kellett MJ, Payne SR. Percutaneous nephrolithotomy: one stage or two? Br J Urol 1984; 56(6):582-5.

[8] Kara C, Resorlu B, Bayindir M, Unsal A.A randomized comparison of totally tubeless and standard percutaneous nephrolithotomy in elderly patients.Urology2010; 76(2):289-93.

[9] Tirtayasa PMW, Yuri P, Birowo P, Rasyid N.Safety of tubeless or totally tubeless drainage and nephrostomy tube as a drainage following percutaneous nephrolithotomy: A comprehens ivereview.Asian J Surg 2017;40(6):419-423.

[10] Pietrow PK, Auge BK, Lallas CD, Santa-Cruz RW, Newman GE,Albala DM, et al. Pain after percutaneous nephrolithotomy: impact of nephrostomy tube size. J Endourol 2003; 17:411e4.

[11] Maheshwari PN, Andankar MG, Bansal M. Nephrostomy tube after percutaneous nephrolithotomy: large-bore or pigtail catheter? J End ourol 2000; 14:735e7. discussion 737e8.

[12] Sivalingam S, Cannon ST, Nakada SY. Current practices in percutaneous nephrolithotomyam ongendourologists. JEndourol 2014; 28:524e7.

[13] Karami H, GholamrezaieHR. Totally tubeless percutaneous nephrolithotomy in selected patients.JEndourol 2004; 18(5):475-6.

[14] Amer T, Ahmed K, Bultitude M, Khan S, Kumar P, De Rosa A, et al. Standard versus tubeless percutaneous nephrolithotomy: a systematic review. UrolInt 2012; 88(4):373-82.

[15] Choi SW, Kim KS, Kim JH, et al. Totally tubeless versus standard percutaneous nephrolithotomy for renal stones: analysis of clinical outcomes and cost. J Endourol 2014; 28:1487e 1494.

[16] Lai WH, Jou YC, Cheng MC, Shen CH, Lin CT, Chen PC, et al. Tubeless percutaneous nephrolithotomy: Experience of 1000 cases at a single institute. Urological science. 2017; 28(1): 23-6

[17] MS, Agrawal M. Tubeless, percutaneous nephrolithotomy. Indian J Urol 2010; 26:16-24.

Citation: Dr. Sachin Dharwadkar, et.al. Tubeless PCNL - Contemporary Analysis of Indications, Safety and Efficacy. ARC Journal of Urology.2019; 4(2):1-5.doi:dx.doi.org/10.20431/2456-060X.0402001.

Copyright: () 2019 Authors. This is an open-access article distributed under the terms of the Creative Commons Attribution License, which permits unrestricted use, distribution, and reproduction in any medium, provided the original author and source are credited. 